be adjusted upon $\epsilon$ of the same constellation, so as to make that perfectly round."

These remarks have an essential bearing upon the investigation of elements. The components must have been very close at both Herschel's epochs-if there be no mistake in the register-and this is not at first sight readily explained by the curve exhibiting the motion of the smaller star from Struve's earliest micrometrical measures in 1825 to the present date.

Herschel further remarked in 1802 that the appearance of the components was much like that of "a planet with a large satellite, or small companion," and strongly suggestive of "the idea of a connection between the two bodies, especially as they are much insulated."

The Rotation of VenUs.-In a note upon the time of rotation and position of the axis of Venus, which recently appeared in this column, reference was inadvertently omitted to Flaugergues' observations at Viviers in July, I796, which, according to a communication from Valz to the Astronomische Nachrichten (No. 278, vol. xi), seemed to favour Bianchini's period, and placed the north pole of Venus in longitude $321^{\circ} 20^{\prime}$, with an elevation of $16^{\circ} 28^{\prime}$. Details of the observations are wanting, but Valz states that Flaugergues observed with "une ancienne lunette à deux verres de 18 pieds de long, amplifiant ro5 fois qu'il dit fort bonne." He also employed one of I 4 feet, and a telescope said to be good, which Legentil brought from India. Valz adds : "J'ai vu le dessein original de la tache, elle etait grande et de forme trapezoide arrondie, \&c."

Hussey's vigorous but prejudiced defence of the extraordinary period of rotation assigned by Bianchini will be found in Astronomische Nachrichten, No 248.

Fritsch, of Quedlinburg, thought some observations of his in April I $80 \mathrm{r}$ indicated a period of $23 \mathrm{~h} .22 \mathrm{~m}$. (Berliner Astronomisches fahrbuch, 1804, p. 213).

\section{SONG OF THE SCREW}

A MOVING form or rigid mass, Under whate'er conditions,

Along successive screws must pass Between each two positions.

It turns around and slides along-

This is the burden of my song.

The pitch of screw, if multiplied By angle of rotation,

Will give the distance it must glide

In motion of translation.

Infinite pitch means pure translation

And zero pitch means pure rotation.

Two motions on two given screws, With amplitudes at pleasure,

Into a third screw-motion fuse;

Whose amplitude we measure

By narallelogram construction

(A very obvious deduction).

Its axis cuts the nodal line

Which to both screws is normal,

And generates a form divine,

Whose name, in language formal,

Is "surface-ruled of third degree."

Cylindroid is the name for me.

Rotation round a given line

Is like a force along.

If to say couple you incline,

You're clearly in the wrong ; -

'Tis obvious, upon reflection,

A line is not a mere direction.
So couples with translations too

In all respects agree ;

And thus there centres in the screw

A wondrous harmony

Of Kinematics and of Statics, -

The sweetest thing in mathematics.

The forces on one given screw,

With motion on a second,

In general some work will do,

Whose magnitude is reckoned

By angle, force, and what we call

The cocfficient virtual.

Rotation now to force convert, And force into rotation ;

Unchanged the work, we can assert, In spite of transformation.

And if two screws no work can claim, Reciprocal will be their name.

Five numbers will a screw define, A screwing motion, six ;

For four will give the axial line,

One more the pitch will fix;

And hence we always can contrive

One screw reciprocal to five.

Screws-two, three, four, or five, combined (No question here of sex),

Yield other screws which are confined Within one screw complex.

Thus we obtain the clearest notion

of ireedom and constraint of motion.

In complex III. three several screws At every point you find,

Or if you one direction choose, One screw is to your mind;

And complexes of order III.

Their own reciprocals may be.

In IV., wherever you arrive,

You find of screws a cone.

On every line in complex $\mathrm{V}$.

There is precisely one;

At each point of this complex rich,

A plane of screws have given pitch.

But time would fail ine to discourse Of Order and Degree,

Of Impulse, Energy, and Force,

And Reciprocity.

All these and more, for motions smail,

Have been discussed by Dr. Ball.

ON THE TELEPHONE, AN INSTRUMENT FOR TRANSMITTING MUSICAL NUTES BY MEANS OF ELECTRICITY

W R. ELISHA GRAY recently read a paper before an American Society explaining his apparatus for transmitting musical notes by electricity. He showed experimentally how, by means of a current of electricity in a single wire, a number of notes could be reproduced simultaneously at a great distance, and how by this means also a number of telegraphic messages could be transmitted at once along a wire and separately received at the other end. One of Mr. Gray's apparatuses was exhibited in London at the last soirêe of the Society of Telegraph Engineers by the president, Mr. Latimer Clark. The principle of the apparatus is as follows :-

A vibrating reed is caused to interrupt the electric current entering the wire a certain number of times per second and the current so interrupted at the sending end sets a similar reed vibrating at the distant end. 
The sending reed is ingeniously maintained in constant vibration by a pair of intermittent electro-magnets which are magnetised and demagnetised by the vibrating reed itself.

Thus in Fig. I (which represents the transmitting part of the telephone and its connections for a single note), the current from the magnet battery flowing in the direction of the small arrow passes through the pair of electromagnets $A$ to the terminal $r$ of the reed $R$, and thence by the spring contact $b$ and the wire $b z$ to the battery again, completing its circuit without passing through the other pair of electro-magnets $B$, which are not therefore magnetised. The reed $R$ is consequently pulled over by the electro-magnets A. But on this taking place the spring contact $b$ is broken and the circuit is no longer completed through $b z$ but through the electro-magnets $B$, which are consequently magnetised, and tend by their induction on the reed to neutralise that of $\mathrm{B}$. The reed

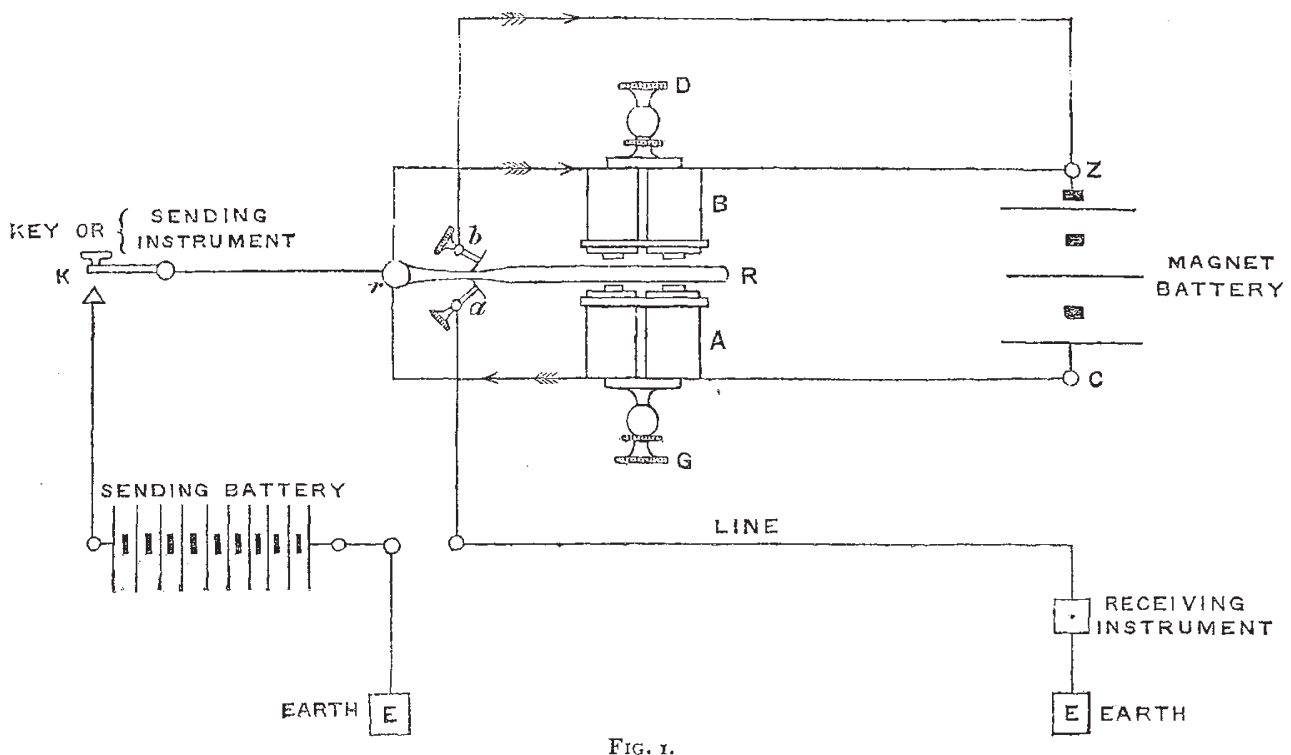

therefore springs back to its intermediary position, but in so doing the contact at $b$ is again made and the electromagnets $B$ again short-circuited and the reed pulled over (or rather assisted over, for it has its own resilience or spring) towards $\mathrm{A}$; so this goes on keeping the reed in vibration berween the electro-magnets and alternately making and breaking the spring contact $b$ and also that of $a$, the number of contacts per second being dependant on the vibrating period of the reed.

1. While this is going on the reed of course emits its musical note. Two Leclanché or bichromate cells are sufficient to work the transmitter and give a good note.

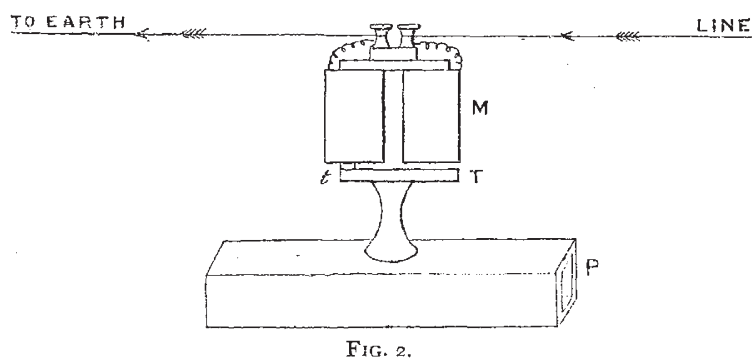

The spring contact $b$ is to be adjusted by the screw there seen until the note emitted by the reed is both loud and pure. The magnets $A$ and $\mathrm{B}$ are adjustable to or from the reed by the milled heads $C$ and $D$.

The spring contact $a$ just mentioned belongs properly to the line circuit. It is the intermittent contact which interrupts the current sent into the line. As will be seen from the diagram the circuit of the sending battery is made through the key $\mathrm{K}$, the reed, and the spring contact $a$. On holding down the key $\mathrm{K}$ the current flows into the line, being interrupted, however, by the contact $a$ as many times per second as the reed vibrates, and this intermittent current flowing to earth at the distant station, $s$ made to elicit a corresponding note from the receiving apparatus there.

The receiving instruments are of two kinds, electromagnetic and physiological.

In the first there is a plain double electro-magnet with a steel tongue having one end rigidly fixed to one pole, the other end being free to vibrate under the other pole. This stands over a wooden pipe closed at one end. Thus in Fig. $2 t \mathrm{~T}$ is the steel tongue fixed at $t$ and free at

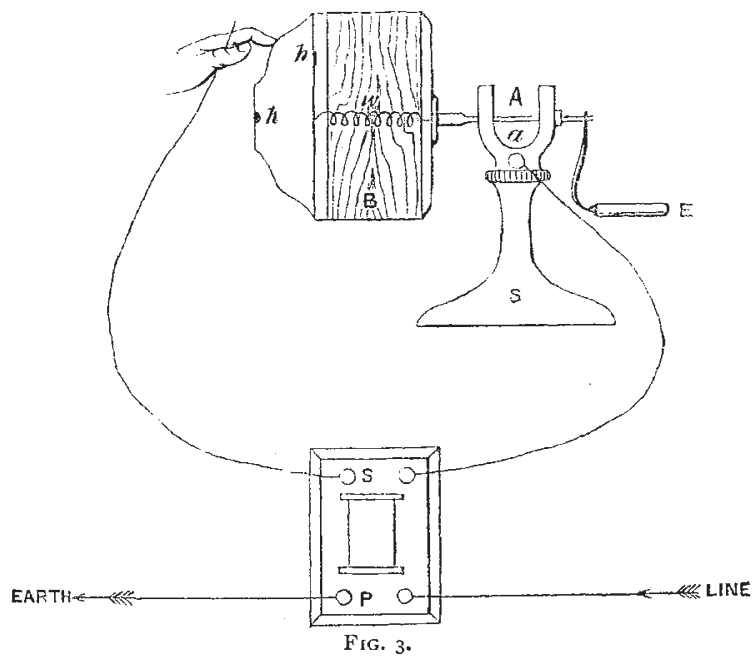

$\mathrm{T}$, while $\mathrm{p}$ is the sounding-pipe. The received current, coming from the line and passing through the electromagnet $M$ to earth, sets the tongue vibrating, and the pipe gives forth the same note as the reed at the sending station. Ten Daniell cells working through I,000 ohms, give a good strong note, especially when the receiver is 
held in the hand close to the head. The screw $a_{3}$ Fig. I, must be adjusted to give the best effect.

The other receiving instrument is the most interesting of the two. It consists of a small induction coil used in conjunction with a peculiar sounding-box, as shown in Fig. 3 .

Here the line-current is passed to earth through the primary circuit $P$ of the small induction coil, and the induced current is led to the sounding-box. This consists of a flat hollow cylindrical wooden box $\mathrm{B}$, covered by a convoluted face of sheet zinc with two air holes $h h$, perforated in it, this box is attached to a metal axle A, turning in forked iron bearings, insulated from but supported by an iron stand S. By this means the soundingbox can be revolved by the ebony handle $\mathrm{E}$. The zinc face is connected across the empty interior of the box by $a$ wire $w$ to the metal bearings on the other side. One end of the secondary circuit of the induction coil is to be connected to the metal bearing by the terminal $a$, and the other to a short bare wire held in the left hand. On then striking a finger of the hand holding the wire smartly across the zinc face, the proper note is sounded by the box; or, what is more convenient, on turning the box by the insulated handle and keeping the point of the finger rubbing on its face, the note is fieard. The rough under side of the finger pressed pretty hard on the bulging part of the face is best. The instant the current is put on by the sending key $K, F i g$. I, the dry rasp of the skin on the zinc-surface becomes changed into a musical note.

These "sounders" can be made to receive indifferently a variety of notes. I have under my care at present a telephone with four transmitters tuned to give the four notes of the common chord, and two receivers, which interpret equally well any one of these notes or all together. But sounders are also made in the same way which will emit only one special note, and so are sensible only to the corresponding current. It is by their means that the telephone can be applied to multiplex telegraphy. As many as eight transmitters may be set to interrupt the line current according to the vibrations of eight different tuning-forks, and the resultant current can be made by means of eight special receivers to reproduce the same number of corresponding notes at the distant station. The current is controlled by eight keys at the sending end and sifted by eight sounders at the receiving end, each sounder being sensitive only to those portions of the current affected by its corresponding transmitter. The superimposed effect of the eight keys and transmitters on the line current can all be separately interpreted at the receiving end. Thus eight messages might be transmitted simultaneously along one wire in the same direction. It would seem hitherto, however, that this method of telegraphy by the telephone is inferior to the ordinary methods in point of speed of signalling, and in the length of circuit which can be worked by a given battery power.

J. MUNRO

OUR PERCEPTION OF THE DIRECTION OF $A$ SOURCE OF SOUND 1

7 HE practical facility with which we recognise the situation of a sounding body has always been rather a thcoretical difficulty. In the case of sight a special optical apparatus is provided whose function it is to modify the uniform excitation of the retina, which a luminous point, wherever situated, would otherwise produce. The mode of action of the crystalline lens of the eye is well understood, and the use of a lens is precisely the device that would at once occur to the mind of an optician ignorant of physiology. The bundle of rays, which would otherwise distribute themselves over the entire retina, and so give no indication of their origin, are I Abstract of 2 Communication to the Musical Association, by Lord
Rayleigh, F.R.S. made to converge upon a single point, whose excitation is to us the sign of an external object in a certain definite direction. If the luminous object is moved, the fact is at once recognised by the change in the point of excitation.

There is nothing in the ear corresponding to the crystalline lens of the eye, and this not accidentally, so to speak, but by the very nature of the case. The efficient action of a lens depends upon its diameter being at least many times greater than the wave-length of light, and for the purposes of sight there is no difficulty in satisfying this requirement. The wave-length of the rays by which we see is not much more than a ten-thousandth part of the diameter of the pupil of the eye. But when we pass to the case of sound and the ear the relative magnitudes of the corresponding quantities are altogether different. The waves of sound issuing from a man's mouth are about eight feet long, whereas the diameter of the passage of the ear is quite small, and could not well have been made a large multiple of eight feet. It is evident therefore that it is useless to look for anything corresponding to the crystalline lens of the eye, and that our power of telling the origin of a sound must be explained in some different way.

It has long been conjectured that the explanation turns upon the combined use of both ears; though but little seems to have been done hitherto in the way of bringing this view to the test. The observations and calculations now brought forward are very incomplete, but may perhaps help to clear the ground, and will have served their pur. pose if they induce others to pursue the subject.

The first experiments were made with the view of finding out with what degree of accuracy the direction of a sound could be determined, and for this it was necessary of course that the observer should have no other materia! for his judgment than that contemplated.

The observer, stationed with his eyes closed in the middle of a lawn on a still evening, was asked to point with the hand in the direction of voices addressed to him by five or six assistants, who continually shifted their position. It was necessary to have several assistants, since it was found that otherwise their steps could be easily followed. The uniform result was that the direction of a human voice used in anything like a natural manner could be told with certainty from a single word, or even vowel to within a few degrees.

But with other sounds the result was different. If the source was on the right or the left of the observer, its position could be told approximately, but it was uncertain whether, for example, a low whistle was in front or behind. This result led us to try a simple sound, such as that given by a fork mounted on a resonance box. It was soon found that whatever might be the case with a truly simple sound, the observer never failed to detect the situation of the fork by the noises accompanying its excitation, whether this was done by striking or by a violin bow. It was therefore necessary to arrange the experiment differently. Two assistants at equal distances and in opposite directions were provided with similar forks and resonators. At a signal given by a fourth, both forks were struck, but only one was held over its resonator, and the observer was asked to say, without moving his head, which he heard. When the observer was so turned that one fork was immediately in front and the other immediately behind, it was impossible for him to tell which fork was sounding, and if asked to say one or the other, felt that he was only guessing. But on turning a quarter round, so as to have one fork on his right and the other on his left, he could tell without fail, and with full confidence in being correct.

The possibility of distinguishing a voice in front from a voice behind would thus appear to depend on the compound character of the sound in a way that it is not easy to understand, and for which the second ear would be of no advantage. But even in the case of a lateral sound 